\title{
Innovative mid-infrared detector concepts
}

\author{
Sven Höfling ${ }^{1,2}$, Andreas Pfenning ${ }^{1}$, Robert Weih ${ }^{1}$, Albert Ratajczak, ${ }^{1}$ Fabian Hartmann, ${ }^{1}$ Georg \\ Knebl, ${ }^{1}$ Martin Kamp, ${ }^{1}$ and Lukas Worschech ${ }^{1}$ \\ ${ }^{1}$ Technische Physik, Physikalisches Institut and Wilhelm Conrad Röntgen-Center for Complex \\ Material Systems, Universität Würzburg, D-97074 Würzburg, Germany \\ ${ }^{2}$ SUPA, School of Physics and Astronomy, University of St Andrews, St Andrews, KY16 9SS, \\ United Kingdom
}

\begin{abstract}
Gas sensing is a key technology with applications in various industrial, medical and environmental areas. Optical detection mechanisms allow for a highly selective, contactless and fast detection. For this purpose, rotational-vibrational absorption bands within the mid infrared (MIR) spectral region are exploited and probed with appropriate light sources. During the past years, the development of novel laser concepts such as interband cascade lasers (ICLs) and quantum cascade lasers (QCLs) has driven a continuous optimization of MIR laser sources. On the other hand side, there has been relatively little progress on detectors in this wavelength range. Here, we study two novel and promising GaSb-based detector concepts: Interband cascade detectors (ICD) and resonant tunneling diode (RTD) photodetectors. ICDs are a promising approach towards highly sensitive room temperature detection of MIR radiation. They make use of the cascading scheme that is enabled by the broken gap alignment of the two binaries GaSb and InAs. The interband transition in $\mathrm{GaSb} / \mathrm{InAs}$-superlattices (SL) allows for normal incidence detection. The cut-off wavelength, which determines the low energy detection limit, can be engineered via the SL period. RTD photodetectors act as low noise and high speed amplifiers of small optically generated electrical signals. In contrast to avalanche photodiodes, where the gain originates from multiplication due to impact ionization, in RTD photodetectors a large tunneling current is modulated via Coulomb interaction by the presence of photogenerated minority charge carriers. For both detector concepts, first devices operational at room temperature have been realized.
\end{abstract}

Keywords: Interband Cascade Detector, Resonant Tunneling Diode, Mid-IR, Gas Sensing

\section{INTRODUCTION}

Just as interband cascade lasers (ICLs) the interband cascade detector (ICD) [1] makes use of the broken gap alignment of the two binaries GaSb and InAs. The conduction band of InAs is situated below the valence band of GaSb which allows for interband tunneling and thus cascading. An ICD stage is composed of three main parts as shown in the band structure in Figure 1. The short period InAs/GaSb superlattice (SL) works as absorption region for MIR radiation. The fact that the effective bandgap in the SL and thus the cutoff wavelength can be tuned via the SL-period introduces great design flexibility while only binary compounds have to be grown. Additionally the interband transition enables normal incidence detection. The absorber region is surrounded by a barrier and an electron relaxation region. On one side electrons are hindered from tunneling back to a previous cascade by an $\mathrm{AlSb} / \mathrm{GaSb}$ electron barrier. This is because the upper level energy level in the absorption region is situated in the bandgap of GaSb. On the other side the AlSb/InAs electron relaxation region enables the transport of electrons to the $\mathrm{InAs} / \mathrm{GaSb}$ interface that is used to connect the cascades in series. Electrons are transferred to the next stage through an interband tunneling process. The cascading scheme helps to overcome the strong coupling between responsivity and diffusion length which plays a major role especially at high temperature operation [2]. In a bulk material the diffusion length is typically shorter than the absorption depth which has a strong impact on the absorption efficiency. By cascading several absorption regions the total thickness of the structure can be designed longer than the diffusion length since the generated carriers only travel through one stage before entering the next one. This helps to improve the detectivity and the signal to noise ratio.

Infrared Remote Sensing and Instrumentation XXIV, edited by Marija Strojnik, Proc. of SPIE Vol. 9973, 997306 · C 2016 SPIE · CCC code: 0277-786X/16/\$18 · doi: 10.1117/12.2237270 


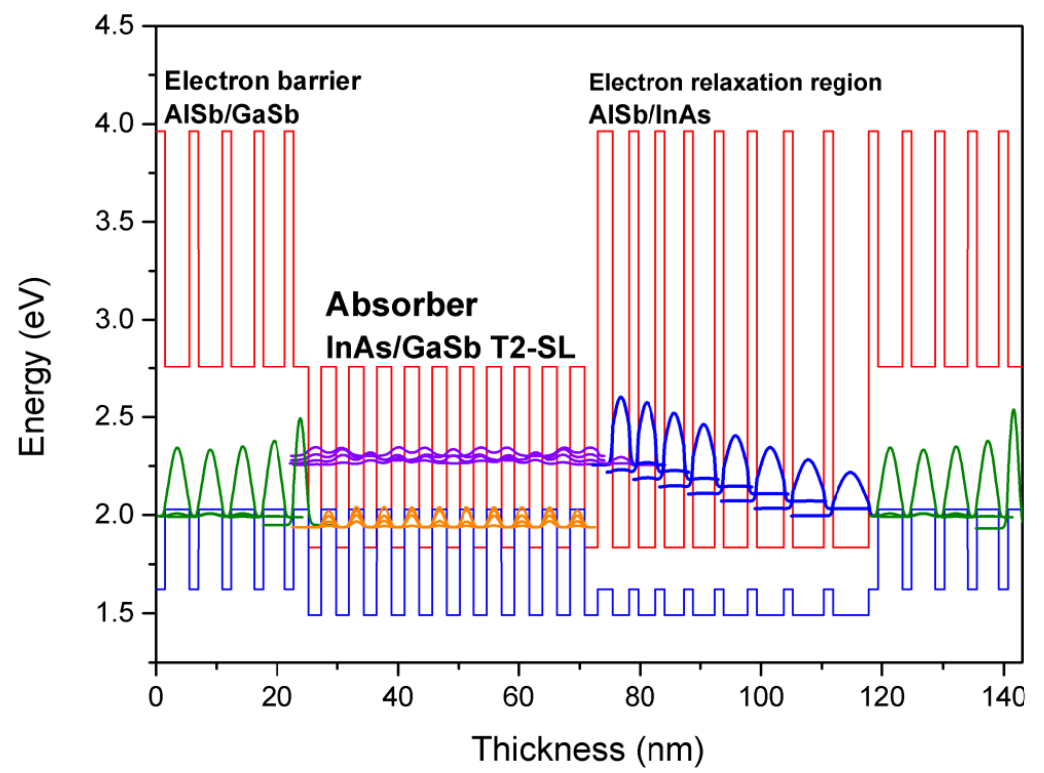

Figure 1: Band structure of one stage of an interband cascade detector (ICD). The main parts are the InAs/GaSb superlattice absorber region, the $\mathrm{AlSb} / \mathrm{GaSb}$ - electron barrier and the AlSb/InAs - electron relaxation region.

The second detector concept for novel MIR-photodetectors discussed in this work is that of GaSb-based resonant tunneling diode photodetectors. Besides being exploited as high-frequency oscillators and emitters in the THz range, [35] Resonant tunneling diodes (RTDs) [6-8] can act as low-noise and high-speed amplifiers of small optically generated electrical signals. [9-11] In contrast to avalanche photodiodes, where the gain originates from multiplication due to impact ionization, in RTD photodetectors a large tunneling current is modulated via Coulomb interaction by the presence of photogenerated minority charge carriers. [12-14] This allows for very high amplification factors of several hundred thousand, [10,11] at considerably small operation voltages. [14,15] The majority of RTD photodetectors has been realized on GaAs or InP based material systems, which allows for excellent light sensing in the visible or near infrared spectral regions, where for example both important telecommunication wavelengths are located. Also based on the InP material system is the similar nano-injector design. $[16,17]$ Nevertheless, when pushing for longer wavelength regions, materials that provide narrower bandgaps need to be considered. Such a material system that provides a huge variety of bandgap energies and heterostructure designs is the so called antimony or $6.1 \AA$ material system, typically based on GaSb substrate growth. [18] We therefore propose to take the RTD principle as it is known from GaAs and InP based systems, and move it to a GaSb-based system. Thus exploiting both, high charge-carrier amplification and low bandgap energies for MIR light detection.

First $\mathrm{GaSb} / \mathrm{AlSb}$ double barrier resonant tunneling diodes with a nearby and lattice-matched GaInAsSb absorption layer for light sensing up to a wavelength of $\lambda=2.76 \mu \mathrm{m}$ have been realized. Their electrical optical properties have been studied by means of electrical transport and photoluminescence measurements, respectively. 


\section{INTERBAND CASCADE DETECTORS}

Since the quality of the InAs/GaSb-SL is of major importance for the performance of the final device careful optimization was done regarding the MBE growth by growing a variety of test samples at different growth conditions. The structures were grown in an Eiko MBE reactor equipped with cracker cells for both As and $\mathrm{Sb}$. The cracking regions of the cracking cells were operated at $950^{\circ} \mathrm{C}$ and $1000^{\circ} \mathrm{C}$, respectively, to ensure efficient cracking of As and $\mathrm{Sb}$. As the structure is grown on GaSb-substrates, the smaller lattice constant of InAs would introduce tensile strain which might result in defect formation or relaxation once the SL reaches a certain thickness. Thus the mean SL lattice constant should match the one of the substrate which was achieved by enforcing InSb interfaces. The 30 period SL test structures were grown at a substrate temperature of $430^{\circ} \mathrm{C}$ and had a nominal SL-period of $4.56 \mathrm{~nm}(2.12 \mathrm{~nm} \mathrm{InAs} / 2.44 \mathrm{~nm} \mathrm{GaSb})$. After each GaSb layer a 2s soak time under Sb flux was applied while the duration of the Sb soak time after the InAs layer was varied between 0 and $6 \mathrm{~s}$. In Figure 2: high resolution X-ray diffraction (HR-XRD) measurements of various samples with soak times of 0,2.1, 3.0 and 6.0 s are shown. The high frequency Pendellösung fringes indicate high material quality for all samples. It can be seen that for the sample with no applied Sb soak time after the InAs layer the 0 th order SL-peak occurs at the right side of the substrate which is a result of tensile strain. With increased soak time the peak shifts further to the left till the SL overall strain becomes compressive due to the InSb - like interfaces. At a soak time of $2.1 \mathrm{~s}$ the mean lattice constant of the SL coincides with the one of the substrate. It should be mentioned that the shift of the SL-peak does not vary linearly with the soak time due to a saturation of the Sb for As exchange reaction [19].
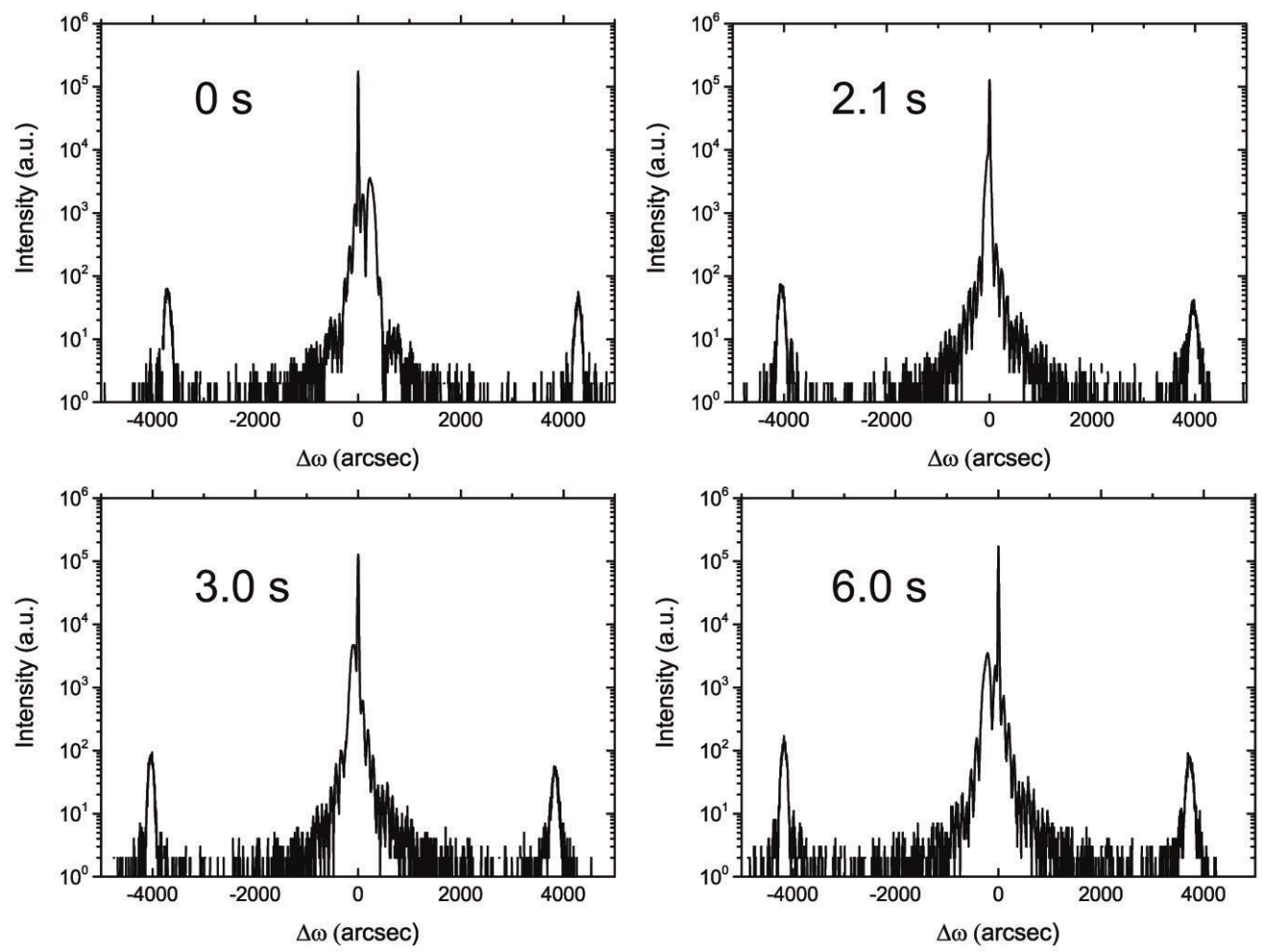

Figure 2: HR-XRD measurement of 30 period InAs/GaSb test samples. The Sb soak time after the InAs layers was varied between $0 \mathrm{~s}$ and $6 \mathrm{~s}$. Strain compensation was achieved for a soak time of $2.1 \mathrm{~s}$.

Furthermore the influence of the growth temperature $\left(380^{\circ} \mathrm{C}-430^{\circ} \mathrm{C}\right)$ on the optical properties of the SL was investigated. Due to the temperature dependence of the Sb for As exchange reaction the $0^{\text {th }}$ order peak of the SL shifted slightly towards smaller angles when the substrate temperature was decreased. No compensation by adjusting the soak time was done in this series of samples. In Figure 3 room temperature photoluminescence (PL) measurements are shown for all samples. The half maximum on the low energy side coincides with the calculated room temperature cutoff 
wavelength of $4.5 \mu \mathrm{m}$. A monotonic increase in PL intensity with decreasing growth temperature was observed. This is a result of higher interface quality which is also confirmed by higher intensity of the SL satellite peaks and more pronounced Pendellösung fringes in HR-XRD measurements. Besides the increase in intensity a shift towards shorter wavelengths is observed for low growth temperatures. This indicates a smaller SL period and could be a result of a change in growth rate with substrate temperature. Taking the PL intensity as a measure of structural quality the test sample grown at the lowest temperature shows the best results. Nevertheless this does not necessarily have to be the optimum growth temperature for an entire ICD structure since the quality of the Al-containing parts of the structure might suffer from low growth temperature.

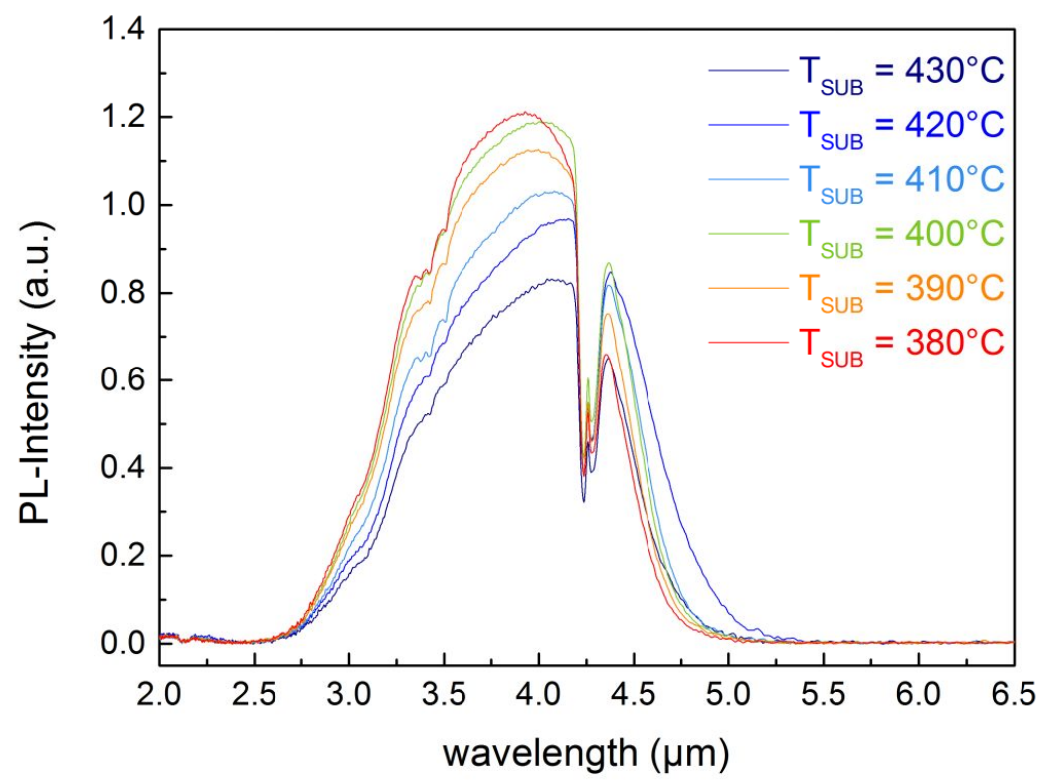

Figure 3: Room temperature PL measurements of SL samples grown at different substrate temperatures. The intensity increases monotonically when the growth temperature is lowered.

Based on the previously shown optimizations an ICD structure was grown by MBE on a p-GaSb substrate. The design incorporated 5 stages with 90 SL periods in each stage. From the grown wafer circular mesas with different diameters in the range from $10 \mu \mathrm{m}$ to $400 \mu \mathrm{m}$ were defined using optical lithography and dry etching. Afterwards a combination of $\mathrm{Si}_{3} \mathrm{~N}_{4}$ and $\mathrm{SiO}_{2}$ was sputtered for sidewall passivation and Ti/Pt/Au-contacts were evaporated. In Figure 4 (a). the IVcharacteristics of devices with different diameters measured at room temperature are shown. At $-0.04 \mathrm{~V}$ the dark current density is as low as $5.2 \times 10^{-4} \mathrm{~A} / \mathrm{cm}^{2}$ for the device with a diameter of $400 \mu \mathrm{m}$ which is comparable to values published in the literature $[20,21]$. In Figure 4 (b) the spectral response curve of the device is shown. One can clearly see the absorption features of $\mathrm{H}_{2} \mathrm{O}$ and $\mathrm{CO}_{2}$. The curve is not corrected for the intensity distribution of the MIR source which explains the slope on the short wavelength side below $2.5 \mu \mathrm{m}$. 

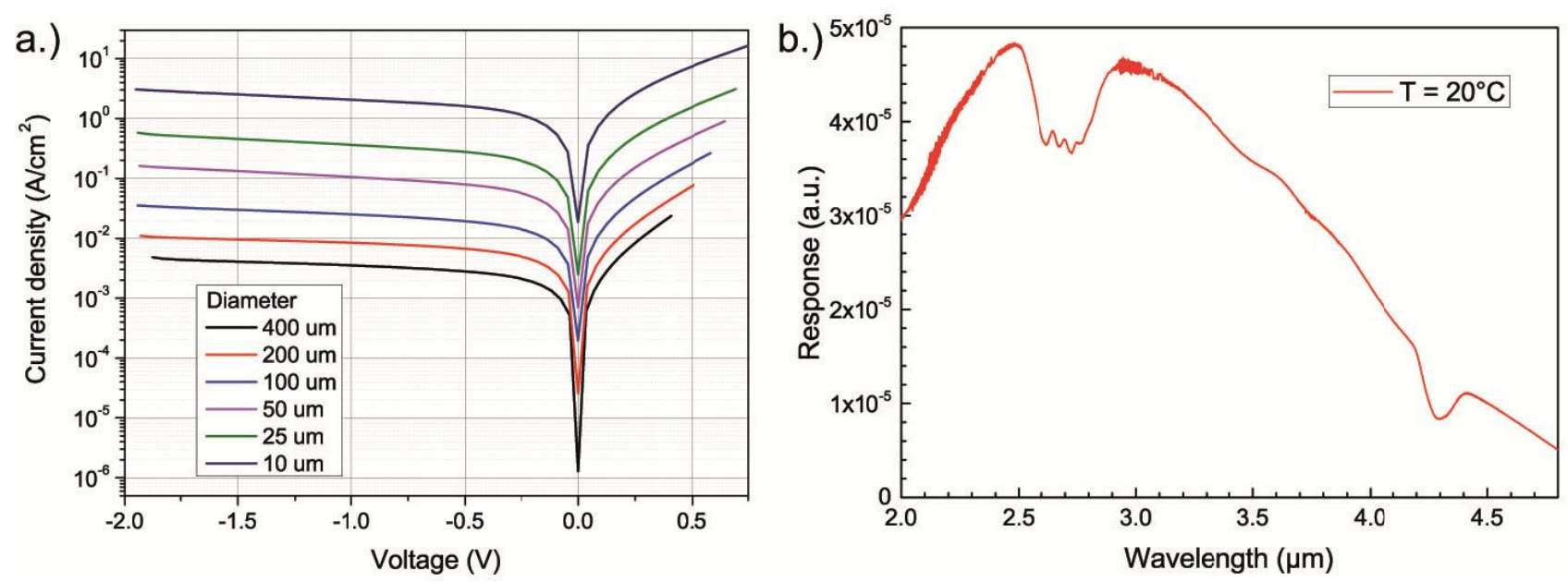

Figure 4: (a) Room temperature current - voltage characteristics of the MIR ICD for different mesa diameters. (b) Room temperature spectral response of the ICD (not normalized for the intensity distribution of the broadband MIR source).

\section{RESONANT TUNNELING BASED DETECTORS}

An example of an RTD photodetector including design and working principle is provided in Figure 5. The device is based on the GaAs material system and was designed for highly sensitive telecommunication light detection at room temperature. With these diodes, sensitivities of above $30 \mathrm{kA} / \mathrm{W}$ have been realized, [11,22] with amplification factors exceeding $3.30 \times 10^{5}$. [15] Figure 5 (a) shows the schematic conduction (CB) and valence band (VB) profile under an applied bias voltage $V$ and under illumination. Figure 5 (b) shows a typical RTD current-voltage characteristic in the dark (black line), and under illumination (red line), calculated after Schulman's model for a physics-based RTD currentvoltage equation. [23] When the RTD is illuminated, incident photons create electron-hole pairs within the GaInNAs absorption region. The thusly photogenerated holes drift towards the AlGaAs/GaAs resonant tunneling structure (RTS), where they accumulate and induce an additional voltage drop across the RTS. [12,13] As a result, the current-voltage characteristics shifts towards lower voltages. [24]

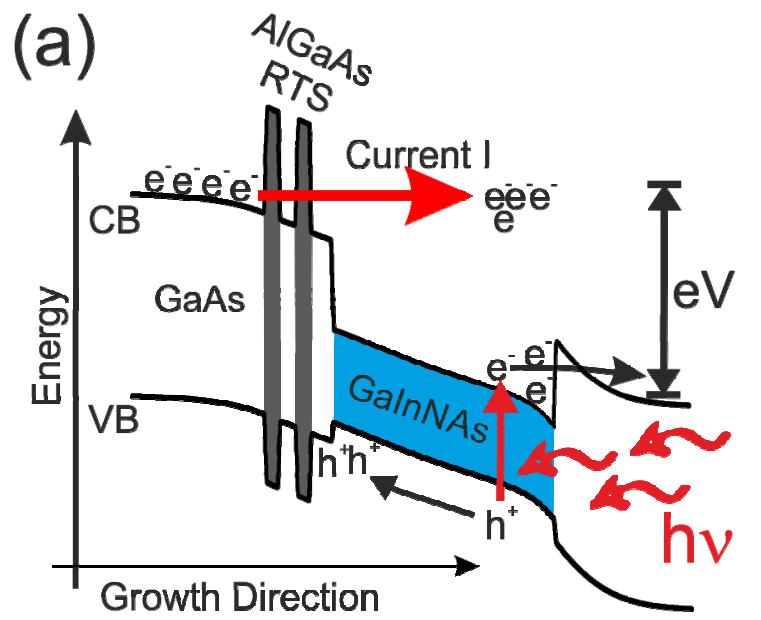

(b)

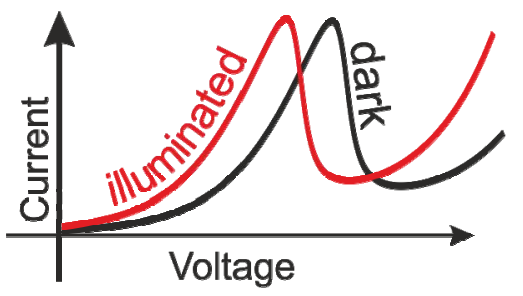

Figure 5: Example of design and working principle of a resonant tunneling diode (RTD) photodetector for room temperature telecommunication light sensing based on the GaAs material system. (a) Schematic conduction (CB) and valence band (VB) profile under an applied positive bias voltage $V$ and under illumination. (b) Sketch of the RTD current-voltage characteristics in the dark (black line) and under illumination (red line). Under illumination, holes are created within the GaInNAs absorption region and drift towards the $\mathrm{AlGaAs} / \mathrm{GaAs}$ resonant tunneling structure (RTS) where they accumulate and induce an additional voltage drop across the RTS. As a result, the current-voltage characteristics under illumination shifts towards lower voltages. 
In order to design and fabricate RTD photodetectors for the mid-infrared spectral region, we propose an approach based on the GaSb material system. Figure 6 (a) shows the conduction (red solid line) and valence band (light blue solid line) profiles of the proposed RTD photodetector underlain with a scanning electron microscopy (SEM) image of the grown structure. The proposed sample was realized by MBE growth on an n-type doped GaSb-substrate. The intrinsic resonant tunneling structure consists of $20 \mathrm{~nm} \mathrm{GaSb}$, and two $4 \mathrm{~nm}$ AlSb barriers sandwiching a $6 \mathrm{~nm} \mathrm{GaSb}$ quantum well. The second AlSb barrier is followed by a $10 \mathrm{~nm} \mathrm{GaSb}$ buffer layer. A slightly n-type doped and $250 \mathrm{~nm}$ wide quaternary GaInAsSb absorption layer with a doping concentration of $n=2.0 \times 10^{17} \mathrm{~cm}^{-3}$ allows for light absorption within the mid-infrared spectral region. The structure is finalized by $300 \mathrm{~nm}$ of highly doped GaSb with $n=3.0 \times 10^{18} \mathrm{~cm}^{-3}$. In the SEM image, dark contrast corresponds to regions with high Al concentration. The two AlSb barriers can be easily identified. Overall, the SEM image confirms excellent crystal quality with highly uniform AlSb barriers, interfaces, and no defects. Figure 6 (b) shows the calculated bandgap energy profile (black line) at room temperature, and the corresponding cut-off wavelength. The bandgap energy of the GaInAsSb absorption layer is $E_{\text {Gap }}=0.451 \mathrm{meV}$, which corresponds to a cut-off wavelength of $\lambda_{C O}=2.76 \mu \mathrm{m}$ (see also Figure 7 (a)). Hence, the RTD will be suited best for light detection within the wavelength range between $\lambda=1.7 \mu \mathrm{m}$ (cut-off wavelength of GaSb) and $\lambda=2.76 \mu \mathrm{m}$. This windows covers important absorption lines of for example water and carbon dioxide.

(a)

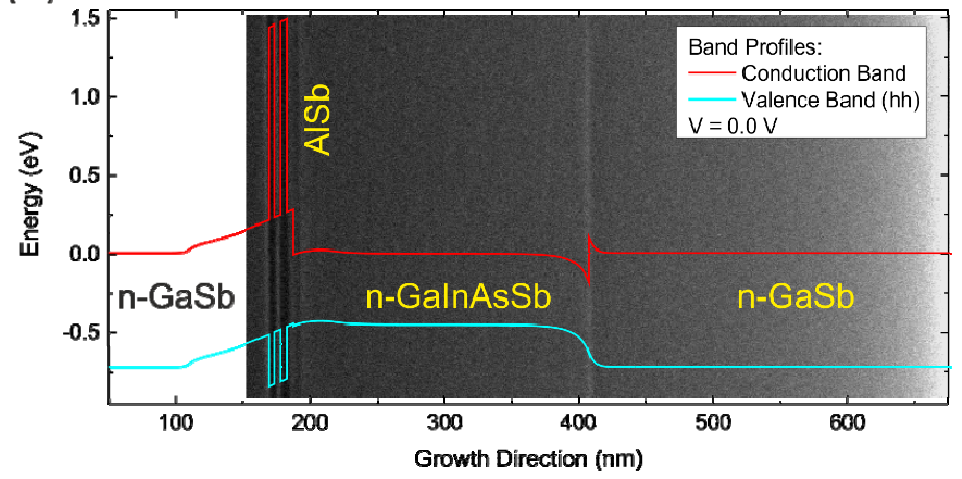

(b)

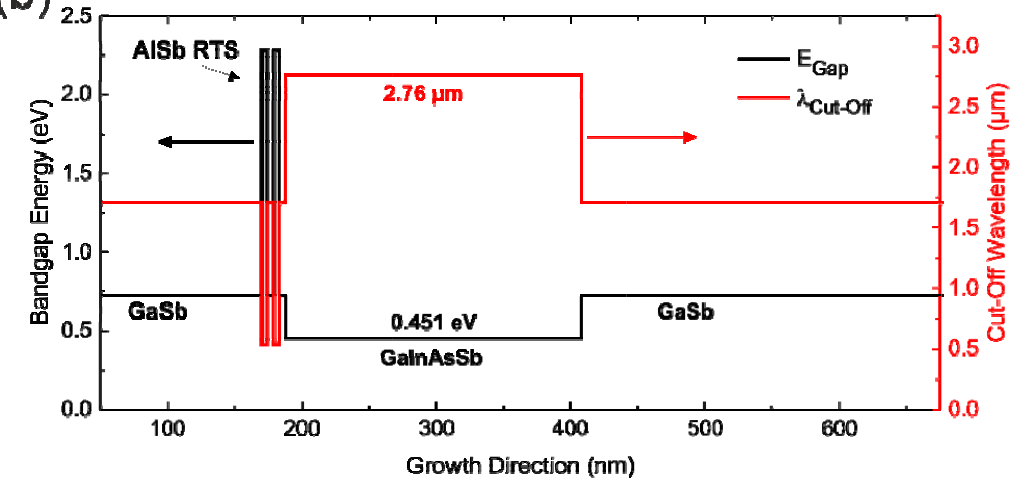

Figure 6: (a) Conduction and valence band profiles of the mid-infrared resonant tunneling diode photodetector. The underlying picture is a scanning electron microscopy image of the grown semiconductor layer structure. Dark contrast represents layers with high Al concentration. The RTD photodetector consists of an undoped double barrier resonant tunneling structure with a nearby and lattice-matched absorption layer. Top and bottom contact layers are formed by highly n-type Te-doped GaSb. The double barrier resonant tunneling structure is made of two $4 \mathrm{~nm}$ thick AlSb barriers sandwiching a $6 \mathrm{~nm}$ GaSb quantum well. The lattice-matched absorption layer is formed by the quaternary semiconductor $\mathrm{Ga}_{0.76} \mathrm{In}_{0.24} \mathrm{As}_{0.20} \mathrm{As}_{0.80}$. The SEM image confirms excellent crystal quality with highly uniform AlSb barriers and interfaces. (b) Room temperature bandgap energy versus crystal position (black line) and the corresponding cut-off wavelength (red line). 
Figure 7 (a) shows the experimental room temperature $\left(T=20^{\circ} \mathrm{C}\right)$ photoluminescence (PL) spectrum of the GaInAsSb absorption layer as solid red line. The PL peak position is located at $\lambda=2.62 \mu \mathrm{m}$. The cut-off wavelength is given by the position of the half-maximum at the low-energy side and located at $\lambda_{C O}=2.76 \mu \mathrm{m}$, which corresponds to a bandgapenergy of $E_{\text {Gap }}=450 \mathrm{meV}$. Figure 7 (b) shows a calculation of the band extrema of the quaternary GaInAsSb lattice matched to GaSb after Ref. [25]. The CB minimum is shown as red spheres, and the VB maximum is shown as black spheres. GaSb CB minimum and VB maximum are indicated by the dashed red and black lines, respectively. At $x=$ 0.24 the bandgap energy is $E_{\text {gap }}=451 \mathrm{meV}$, which perfectly fits the PL measurement.

(a)

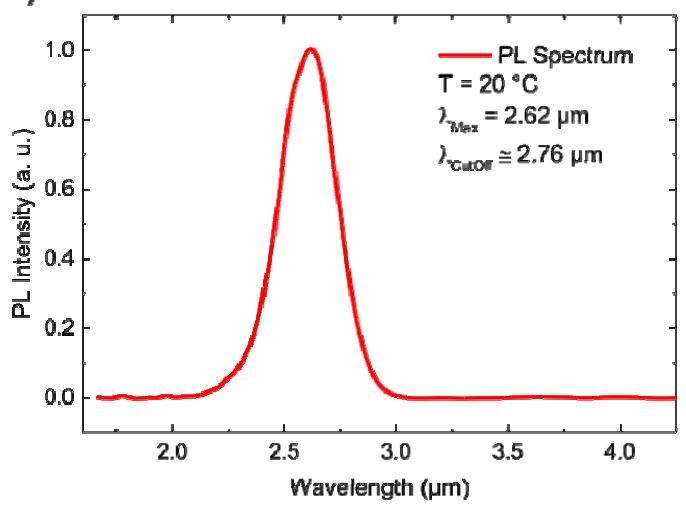

(b)

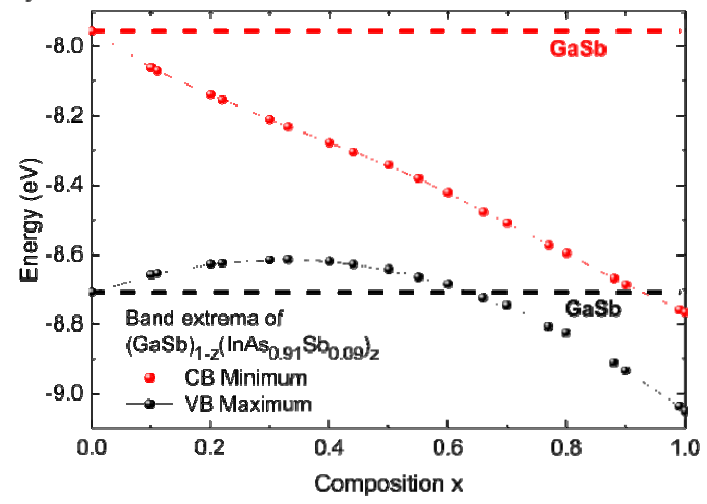

Figure 7: (a) Experimental room temperature photoluminescence (PL) spectrum of the GaInAsSb absorption layer. The PL peak position is located at $\lambda=2.62 \mu \mathrm{m}$. The cut-off wavelength is given by the position of the half-maximum at the lowenergy side and located at $\lambda_{\text {CutOff }}=2.76 \mu \mathrm{m}$. The cut-off wavelength corresponds to a bandgap-energy of $E_{G a p}=450$ meV. (b) Energy position of the GaInAsSb conduction band (CB) minimum and valence band (VB) maximum for latticematched growth condition as a function of mole-fraction composition x. At $x=0.24$ the bandgap energy is $E_{g a p}=451$ meV. CB minimum and VB maximum are calculated according to Ref. [25].

Figure 8 depicts the experimental RTD current density-voltage $(\mathrm{j}(\mathrm{V})-)$ characteristics taken at three different temperatures. The $\mathrm{j}(\mathrm{V})$-characteristic for $T=4.2 \mathrm{~K}$ is shown as blue spheres. A well-pronounced current bistability is found with a peak current-density of $j_{\text {peak }}=3.73 \mu \mathrm{A} / \mu \mathrm{m}^{2}$ at $V=1.92 \mathrm{~V}$, and a valley current density of $j_{\text {val }}=0.33$ $\mu \mathrm{A} / \mu \mathrm{m}^{2}$ at $V=1.88 \mathrm{~V}$. The corresponding peak-to-valley current ratio is $P V R=11.3$. According to Jimenez et al, the current bistability is of intrinsic nature (see Ref. [26,27]). Other than that, there is little literature on that topic.

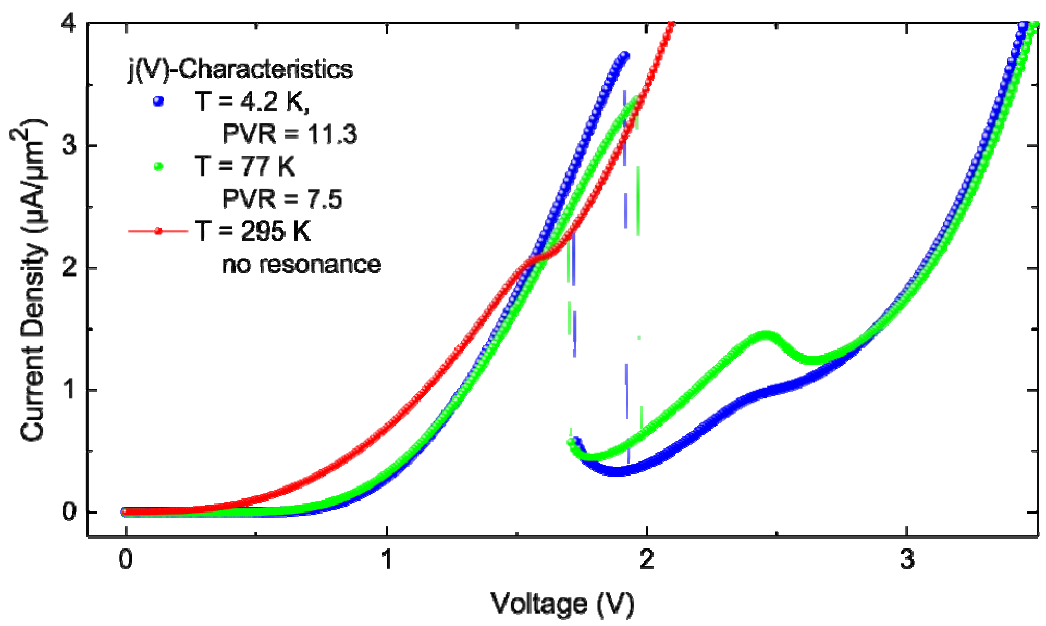

Figure 8: Current density-voltage $(\mathrm{j}(\mathrm{V})-)$ characteristics measured at three different temperatures: submerged in liquid Helium $(T=4.2 \mathrm{~K}$, blue spheres); submerged in liquid nitrogen $(T=77 \mathrm{~K}$, green spheres); and at room temperature ( $T \cong 295 \mathrm{~K}$, red spheres). Well-pronounced current resonances can be observed at $T=4.2 \mathrm{~K}$ and $T=77 \mathrm{~K}$ with peak-tovalley current ratios of $P V R=11.3$ and $P V R=7.5$, respectively. At room temperature, resonant tunneling cannot be observed. 
When increasing the temperature up to $T=77 \mathrm{~K}$ (green circles), the peak current density decreases down to $j_{\text {peak }}=$ $3.38 \mu \mathrm{A} / \mu \mathrm{m}^{2}$, whereas the valley current density increases to $j_{v a l}=0.45 \mu \mathrm{A} / \mu \mathrm{m}^{2}$, with $P V R=7.5$. Decrease of peak and increase of valley current density with increasing temperature can be attributed to two different mechanisms. On the one hand side, at higher temperatures increased phonon scattering reduces the number of electrons that can tunnel resonantly, and increases the number of electrons contributing to non-coherent transport. On the other hand, increasing the temperature leads to a depopulation of electrons in the $\mathrm{GaSb} \Gamma$-valley, while increasing the electron population of the L-valley states. Hence, less electrons can contribute to tunneling via the $\Gamma$ - $\Gamma$ tunneling path. [28] At room temperature, no region of negative differential conductance, and therefore no resonant tunneling can be observed. We are currently investigating different approaches on how to improve the electrical transport properties of GaSb-based resonant tunneling diodes at room temperature. [29]

\section{CONCLUSIONS}

Two promising, novel and alternative detector concepts for the MIR region are demonstrated. ICDs combine the advantages of conventional type-II SL-detectors with the cascading scheme of ICLs. By applying proper soak times during the InAs/GaSb-SL growth the mean lattice constant of the SL was matched to the GaSb substrate. Furthermore a series of samples under variation of the substrate temperature was grown. The highest room temperature PL intensity was observed for the sample grown at the lowest substrate temperature $\left(380^{\circ} \mathrm{C}\right)$. Finally a full ICD structure was grown and processed into circular detector devices operational at room temperature. The cut off wavelength was around $4.5 \mu \mathrm{m}$. Additionally a resonant tunneling diode photodetector with cut-off wavelength at $\lambda=2.76 \mu \mathrm{m}$ is presented. It is based on an $\mathrm{AlSb} / \mathrm{GaSb}$ double barrier resonant tunneling structure with a nearby and lattice-matched GaInAsSb-absorption

layer. First results are promising, but indicate that for room temperature operation further approaches to improve room temperature resonant tunneling need to be considered.

\section{ACKNOWLEDGEMENTS}

The authors are grateful for financial support by the state of Bavaria, the German Ministry of Education and Research (BMBF) within the national project HIRT (FKZ 13XP5003B). Expert technical assistance by M. Emmerling and A. Wolf is gratefully acknowledged. 


\section{REFERENCES}

1. R. Q. Yang, Z. Tian, Z. Cai, J. F. Klem, M. B. Johnson, and H. C. Liu, "Interband-cascade infrared photodetectors with superlattice absorbers," J. Appl. Phys. 107, 054514 (2010).

2. P. Martyniuk, J. Antoszewski, M. Martyniuk, L. Faraone, and A. Rogalski, "New concepts in infrared photodetector designs," Appl. Phys. Rev. 1, 041102 (2014).

3. T. Maekawa, H. Kanaya, S. Suzuki, and M. Asada, "Oscillation up to $1.92 \mathrm{THz}$ in resonant tunneling diode by reduced conduction loss," Appl. Phys. Express 9, 024101 (2016).

4. S. Suzuki, M. Asada, A. Teranishi, H. Sugiyama, and H. Yokoyama, "Fundamental oscillation of resonant tunneling diodes above $1 \mathrm{THz}$ at room temperature," Appl. Phys. Lett. 97, 33-36 (2010).

5. M. Feiginov, H. Kanaya, S. Suzuki, and M. Asada, "Operation of resonant-tunneling diodes with strong back injection from the collector at frequencies up to $1.46 \mathrm{THz}, "$ Appl. Phys. Lett. 104, (2014).

6. R. Tsu and L. Esaki, "Tunneling in a finite superlattice," Appl. Phys. Lett. 22, 562 (1973).

7. L. L. Chang, L. Esaki, and R. Tsu, "Resonant tunneling in semiconductor double barriers," Appl. Phys. Lett. 24, 593 (1974).

8. G. Haddad, P. Mazumder, and J. N. Schulman, "Resonant tunneling diodes: models and properties," Proc. IEEE 86, 641-660 (1998).

9. B. Romeira, L. M. Pessoa, H. M. Salgado, C. N. Ironside, and J. M. L. Figueiredo, "Photo-detectors integrated with resonant tunneling diodes.," Sensors (Basel). 13, 9464-82 (2013).

10. W. Wang, Y. Hou, D. Xiong, N. Li, W. Lu, W. Wang, H. Chen, J. Zhou, E. Wu, and H. Zeng, "High photoexcited carrier multiplication by charged InAs dots in AlAs/GaAs/AlAs resonant tunneling diode," Appl. Phys. Lett. 92, 023508 (2008).

11. A. Pfenning, F. Hartmann, F. Langer, S. Höfling, M. Kamp, and L. Worschech, "Cavity-enhanced resonant tunneling photodetector at telecommunication wavelengths," Appl. Phys. Lett. 104, 101109 (2014).

12. P. England, J. E. Golub, L. T. Florez, and J. P. Harbison, "Optical switching in a resonant tunneling structure," Appl. Phys. Lett. 58, 887 (1991).

13. P. W. Park, H. Y. Chu, S. G. Han, Y. W. Choi, G. Kim, and E.-H. Lee, "Optical switching mechanism based on charge accumulation effects in resonant tunneling diodes," Appl. Phys. Lett. 67, 1241 (1995).

14. A. Pfenning, F. Hartmann, M. Rebello Sousa Dias, F. Langer, M. Kamp, L. K. Castelano, V. Lopez-Richard, G. E. Marques, S. Höfling, and L. Worschech, "Photocurrent-voltage relation of resonant tunneling diode photodetectors," Appl. Phys. Lett. 107, 081104 (2015).

15. A. Pfenning, F. Hartmann, F. Langer, M. Kamp, S. Höfling, and L. Worschech, "Sensitivity of resonant tunneling diode photodetectors," Nanotechnology 27, 355202 (2016).

16. O. G. Memis, A. Katsnelson, S. C. Kong, H. Mohseni, M. Yan, S. Zhang, T. Hossain, N. Jin, and I. Adesida, "A photon detector with very high gain at low bias and at room temperature," Appl. Phys. Lett. 91, 6-9 (2007).

17. V. Fathipour, S. J. Jang, I. H. Nia, and H. Mohseni, "Impact of three-dimensional geometry on the performance of isolated electron-injection infrared detectors," Appl. Phys. Lett. 106, 021116 (2015).

18. H. Kroemer, "The 6.1 family (InAs, GaSb, AlSb) and its heterostructures: a selective review," Phys. E Lowdimensional Syst. Nanostructures 20, 196-203 (2004).

19. M. Losurdo, P. Capezzuto, G. Bruno, A. S. Brown, T. Brown, and G. May, "Fundamental reactions controlling anion exchange during mixed anion heterojunction formation: Chemistry of As-for-Sb and Sb-for-As exchange reactions," J. Appl. Phys. 100, 013531 (2006).

20. Z.-B. Tian, T. Schuler-Sandy, and S. Krishna, "Electron barrier study of mid-wave infrared interband cascade photodetectors," Appl. Phys. Lett. 103, 083501 (2013). 
21. Zhao-Bing Tian and S. Krishna, "Mid-Infrared Interband Cascade Photodetectors With Different Absorber Designs," IEEE J. Quantum Electron. 51, 1-5 (2015).

22. A. Pfenning, F. Hartmann, F. Langer, M. Kamp, S. Höfling, and L. Worschech, "Cavity-enhanced AlGaAs/GaAs resonant tunneling photodetectors for telecommunication wavelength light detection at $1.3 \mu \mathrm{m}$," in Proc. SPIE 9608, Infrared Remote Sensing and Instrumentation XXIII, M. Strojnik Scholl and G. Páez, eds. (2015), Vol. 9608, p. 960810.

23. J. Schulman, H. J. De Los Santos, and D. H. Chow, "Physics-based RTD current-voltage equation," IEEE Electron Device Lett. 17, 220-222 (1996).

24. I. J. S. Coêlho, J. F. Martins-Filho, J. M. L. Figueiredo, and C. N. Ironside, "Modeling of light-sensitive resonant-tunneling-diode devices," J. Appl. Phys. 95, 8258 (2004).

25. K. Shim, "Composition dependence of band alignments in Ga[sub $\mathrm{x}] \operatorname{In}[\operatorname{sub} 1-\mathrm{x}] \mathrm{As}[\operatorname{sub} \mathrm{y}] \mathrm{Sb}[\operatorname{sub} 1-\mathrm{y}]$ heterojunctions lattice matched to GaSb and InAs," J. Appl. Phys. 114, 203703 (2013).

26. J. Jimenez, E. Mendez, X. Li, and W. Wang, "Intrinsic bistability by charge accumulation in an L-valley state in GaSb-AlSb resonant-tunneling diodes," Phys. Rev. B 52, (1995).

27. J. Jimenez, E. Mendez, X. Li, and W. Wang, "Resonant tunneling and intrinsic bistability in GaSb-based double barrier heterostructures," Solid. State. Electron. 40, 583-584 (1996).

28. J. L. Jimenez, X. Li, and W. I. Wang, "Resonant tunneling in AlSb-GaSb-AlSb and AlSb-InGaSb-AlSb double barrier heterostructures," Appl. Phys. Lett. 64, 2127 (1994).

29. A. Pfenning, G. Knebl, F. Hartmann, R. Weih, A. Bader, M. Emmerling, S. Höfling, and L. Worschech, "In Preparation: Room temperature operation of GaSb-based resonant tunneling diodes due to enhanced prewell injection," (2016). 